PROCEEDINGS OF THE

AMERICAN MATHEMATICAL SOCIETY

Volume 138, Number 10, October 2010, Pages 3531-3539

S 0002-9939(2010)10277-0

Article electronically published on June 15, 2010

\title{
ON SPECTRAL GAP RIGIDITY AND CONNES INVARIANT $\chi(M)$
}

\author{
SORIN POPA \\ (Communicated by Marius Junge)
}

\begin{abstract}
We calculate Connes' invariant $\chi(M)$ for certain $\mathrm{II}_{1}$ factors $M$ that can be obtained as inductive limits of subfactors with spectral gap. Then we use this to answer a question he posed in 1975 on the structure of McDuff factors $M$ with $\chi(M)=1$.
\end{abstract}

\section{INTRODUCTION}

Given a $\mathrm{II}_{1}$ factor $M$, one denotes by $\chi(M)$ the image in the outer automorphism $\operatorname{group} \operatorname{Out}(M)=\operatorname{Aut}(M) / \operatorname{Int}(M)$ of the group of automorphisms of $M$ that are both approximately inner and centrally free. This invariant for $\mathrm{II}_{1}$ factors was introduced by Connes in [2, who used it to solve several famous problems in von Neumann algebras. On this occasion, he raised some questions on $\chi(M)$, one of them being whether a $M c D$ uff factor (i.e. a $\mathrm{II}_{1}$ factor that splits off the hyperfinite $\mathrm{II}_{1}$ factor $R$ ) with $\chi(M)=1$ is necessarily of the form $M=Q \bar{\otimes} R$ with $Q$ a nonGamma $\mathrm{II}_{1}$ factor; i.e., in the terminology used hereafter, $M$ is $s$-McDuff (strong McDuff) 1

We answer this question here by providing several classes of examples of McDuff factors who have trivial $\chi(M)$ but are not s-McDuff. We do this by using the deformation-rigidity methods in [17, 18, 19. Thus, we first show that any infinite tensor product of non-Gamma $\mathrm{II}_{1}$ factors, $M=\bar{\otimes}_{n} Q_{n}$, satisfies these properties. The second class of examples comes from subfactor theory. Thus, starting from a non-trivial irreducible inclusion of non-Gamma factors $P \subset N$ with finite depth, we consider the enveloping factor $N_{\infty}$, obtained as the inductive limit of the associated Jones tower of factors, $P \subset N \subset N_{1} \subset N_{2} \subset \ldots$. We then use 14] to show that if the graph $\Gamma=\Gamma_{P, N}$ of $P \subset N$ has the property that the Perron-Frobenius eigenvector $\vec{s}$ (resp. $\vec{t}$ ) of $\Gamma^{t} \Gamma$ (resp. $\Gamma \Gamma^{t}$ ) has distinct entries (e.g. if $\Gamma_{P, N}=A_{2 n}$ ), then $\chi\left(N_{\infty}\right)=1$. On the other hand, by using deformation-rigidity we prove that $N_{\infty}$ is not s-McDuff.

We mention that the deformation of the ambient factors that we use in our arguments is by inductive limits, while the rigidity part is played by the spectral

Received by the editors September 30, 2009 and, in revised form, October 25, 2009 and October $31,2009$.

2000 Mathematics Subject Classification. Primary 46L10, 46L37, 46L40.

This work was supported in part by NSF Grant 0601082.

${ }^{1}$ I am grateful to V.F.R. Jones for bringing Connes' question to my attention. 
gap property, considered in [19. More applications of these ideas and techniques can be found in $[20$.

\section{Spectral gap and automorphisms in $\operatorname{Ctr}(M), \overline{\operatorname{Int}(M)}$}

2.1. Definition. Let $M$ be a $\mathrm{II}_{1}$ factor and let $Q \subset M$ be a subfactor. We say that $Q$ has spectral gap in $M$ if $\forall \varepsilon>0, \exists u_{1}, \ldots, u_{n} \in \mathcal{U}(Q)$ and $\delta>0$ such that if $x \in M$ satisfies $\left\|\left[x, u_{i}\right]\right\|_{2} \leq \delta\|x\|_{2}, \forall i$, then $\left\|x-E_{Q^{\prime} \cap M}(x)\right\|_{2} \leq \varepsilon\|x\|_{2}$.

The following lemma relates this property with a somewhat weaker version of spectral gap, useful in applications. As usual, we denote by $\omega$ a free ultrafilter on $\mathbb{N}$ and by $M^{\omega}$ the ultrapower of $M$ (itself a $\mathrm{II}_{1}$ factor), as defined in [8].

2.2. Lemma. If $Q \subset M$ is an inclusion of factors and $Q$ has spectral gap in $M$, then $Q^{\prime} \cap M^{\omega}=\left(Q^{\prime} \cap M\right)^{\omega}$.

Proof. Trivial by the definitions.

2.3. Lemma. Assume the $\mathrm{II}_{1}$ factor $M$ is an inductive limit of subfactors $N_{n} \nearrow M$ which have spectral gap in $M$. Then $M^{\prime} \cap M^{\omega}=\bigcap_{n}\left(N_{n}^{\prime} \cap M\right)^{\omega}=\left\{\left(x_{n}\right) \mid \exists k_{n}\right.$ such that $x_{n} \in N_{k_{n}}^{\prime} \cap M$ and $\left.\lim _{\omega} k_{n}=\infty\right\}$.

Proof. To see the first equality, note that we have $M^{\prime} \cap M^{\omega}=\left(\bigcup_{n} N_{n}\right)^{\prime} \cap M^{\omega}=$ $\bigcap_{n}\left(N_{n}^{\prime} \cap M^{\omega}\right)$, which by Lemma 2.2 is equal to $\bigcap_{n}\left(N_{n}^{\prime} \cap M\right)^{\omega}$.

To show the second equality, denote by $\mathcal{Y}$ the given set. We clearly have $\mathcal{Y} \subset$ $\bigcap_{n}\left(N_{n}^{\prime} \cap M\right)^{\omega}$. Conversely, if $\left(x_{n}\right)_{n} \in \bigcap_{m}\left(N_{m}^{\prime} \cap M\right)^{\omega}$, then for any $m$ there exists a neighborhood $V_{m}$ of $\omega$ such that $\left\|x_{k}-E_{N_{m}^{\prime} \cap M}\left(x_{k}\right)\right\|_{2} \leq 2^{-m}, \forall k \in V_{m}$. Moreover, we can take $V_{m} \subset V_{m-1}$. Thus, if for each $n \in V_{m} \backslash V_{m-1}$ we denote $y_{n}=E_{N_{m}^{\prime} \cap M}\left(x_{n}\right)$ and put $k_{n}=m$, then $\left(y_{n}\right)_{n}$ coincides with $\left(x_{n}\right)_{n}$ in $M^{\omega}$ and clearly $y \in \mathcal{Y}$ and $k_{n}$ satisfy the required conditions.

2.4. Lemma. Assume that $Q \subset P$ is an inclusion of factors. Let $0<\varepsilon<1 / 2$ and set $\delta=(\varepsilon / 6)^{8}$. If $\theta \in \operatorname{Aut}(P)$ satisfies $\|\theta(v)-v\|_{2} \leq \delta, \forall v \in \mathcal{U}(Q)$, then there exists a partial isometry $w \in \mathcal{U}(P)$ such that $\theta(x) w=w x, \forall x \in Q$, and $\|w-1\|_{2} \leq \varepsilon$. If in addition $Q^{\prime} \cap P$ is a factor, then there exists $u \in \mathcal{U}(P)$ such that $u$ still satisfies $\theta(x) u=u x, \forall x \in Q$, and $\|u-1\|_{2} \leq 2 \varepsilon$.

Proof. Let $K=\overline{\mathrm{co}}^{w}\left\{\theta(v) v^{*} \mid \mathcal{U}(Q)\right\}$. Note that $\|y\| \leq 1$ and $\|y-1\|_{2} \leq \delta$, for all $y \in K$. Since $K$ is convex and weakly compact in $P \subset L^{2}(P)$, there exists a unique element $\xi$ of minimal norm $\|\cdot\|_{2}$ in $K$. Since $\theta(v) \xi v^{*} \in K$ and $\left\|\theta(v) \xi v^{*}\right\|_{2}=\|\xi\|_{2}$, $\forall v \in U(Q)$, by the uniqueness of $\xi$ it follows that $\theta(x) \xi=\xi x, \forall x \in Q$. Thus, the partial isometry $w \in P$ in the polar decomposition of $\xi$ still satisfies $\theta(x) w=w x$, $\forall x \in Q$. Moreover, by [3] we have $\|w-1\|_{2} \leq 6 \delta^{1 / 8}=\varepsilon$.

If in addition $Q^{\prime} \cap P$ is a factor and we assume $\varepsilon<1 / 2$, then $\tau\left(w w^{*}\right) \geq 1 / 2$ and we can take partial isometries $e_{12} \in Q^{\prime} \cap P, f_{21} \in \theta(Q)^{\prime} \cap P$ such that $e_{12} e_{12}^{*} \leq w^{*} w$, $e_{12}^{*} e_{12}+w^{*} w=1, f_{21}^{*} f_{21}=w e_{12} e_{12}^{*} w^{*}, f_{21} f_{21}^{*}+w w^{*}=1$. Thus, if we define $u=$ $w+f_{21} w e_{12}$, then $u$ is a unitary satisfying $\theta(x) u=u x, \forall x \in Q$, and $\|u-1\|_{2} \leq 2 \varepsilon$, proving the statement.

2.5. Lemma. Let $\theta \in \operatorname{Aut}(M),\left\{u_{n}\right\}_{n} \subset U(M)$. Then we have:

(a) If $N_{0} \subset M$ has spectral gap and $\theta(x)=\lim _{n} u_{n} x u_{n}^{*}, \forall x \in M$, then

$$
\lim _{n, m \rightarrow \infty}\left\|u_{n}^{*} u_{m}-E_{N_{0}^{\prime} \cap M}\left(u_{n}^{*} u_{m}\right)\right\|_{2}=0 .
$$


If in addition $\theta_{\mid N_{0}}=i d$, then there exist $v_{n} \in \mathcal{U}\left(N_{0}^{\prime} \cap M\right)$ such that $\theta(x)=$ $\lim _{n} v_{n} x v_{n}^{*}, \forall x \in M$.

(b) Assume $M$ is an inductive limit of subfactors $N_{n} \nearrow M$ with spectral gap such that $N_{k}^{\prime} \cap M$ is a factor, $\forall k \geq 0$. Then $\theta \in \overline{\operatorname{Int}(M)}$ iff $\exists v_{k} \in \mathcal{U}(N)$ such that $\theta_{\mid N_{k}}=\operatorname{Ad}\left(v_{k}\right)_{\mid N_{k}}, \forall k \geq 0$. Moreover, if this is the case, then $U_{n}=v_{0}^{*} v_{n} \in N_{0}^{\prime} \cap M$ and we have $\theta(x)=\operatorname{Ad} v_{0} \lim _{n} \operatorname{Ad} U_{n}(x), \forall x \in M$.

Proof. The first part of $(a)$ is trivial. To prove the second part, note that the spectral gap of $N_{0}$ in $M$ and the condition $\lim _{n} u_{n} x u_{n}^{*}=x, \forall x \in N_{0}$, imply $\lim _{n}\left\|u_{n}-E_{N_{0}^{\prime} \cap M}\left(u_{n}\right)\right\|_{2}=0$. By [3], there exist unitary elements $v_{n} \in \mathcal{U}\left(N_{0}^{\prime} \cap M\right)$ such that $\lim _{n}\left\|v_{n}-E_{N_{0}^{\prime} \cap M}\left(u_{n}\right)\right\|_{2}=0$, and thus $\lim _{n}\left\|u_{n}-v_{n}\right\|_{2}=0$ as well, showing that $\theta=\lim \operatorname{Ad}\left(v_{n}\right)$. To prove part (b), assume $\theta=\lim _{n} \operatorname{Ad}\left(u_{n}\right)$, fix $k \geq 1$ and let $n_{0}$ be such that $\left\|u_{n}^{*} u_{m}-E_{N_{k}^{\prime} \cap M}\left(u_{n}^{*} u_{m}\right)\right\|_{2} \leq 12^{-8}, \forall n, m \geq n_{0}$ (by $(a)$ ). Then, for $x \in \mathcal{U}\left(N_{k}\right)$ we have $u_{n_{0}}^{*} \theta(x) u_{n_{0}}=\lim _{m \rightarrow \infty} u_{n_{0}}^{*} u_{m} x u_{m}^{*} u_{n_{0}}$ and thus

$$
\left\|u_{n_{0}}^{*} \theta(x) u_{n_{0}}-x\right\|_{2} \leq 2 \underset{m}{\limsup }\left\|u_{n_{0}}^{*} u_{m}-E_{N_{k}^{\prime} \cap M}\left(u_{n_{0}}^{*} u_{m}\right)\right\|_{2} \leq 2 \cdot 12^{-8} .
$$

But this implies $\operatorname{Ad}\left(u_{n_{0}}^{*}\right) \circ \theta$ is inner on $N_{k}$, by Lemma 2.4. Thus, there exists $V_{k} \in \mathcal{U}(M)$ such that $v_{k}=u_{n_{0}} V_{k}$ satisfies $\theta(x)=\operatorname{Ad} v_{k}, \forall x \in N_{k}$.

2.6. Lemma. Assume $N_{n} \nearrow M, N_{n}$ has spectral gap in $M$ and $N_{n}^{\prime} \cap M$ is a factor, $\forall n$. Then $\theta \in \operatorname{Ctr}(M)$ iff there exist $n$ and $u \in \mathcal{U}(M)$ such that $\theta_{\mid N_{n}^{\prime} \cap M}=\operatorname{Ad}(u)$.

Proof. By Lemma 2.3, if there exist $n$ and $u \in \mathcal{U}(M)$ such that $\theta(x)=u x u^{*}$, $\forall x \in N_{n}^{\prime} \cap M$, then $\theta \in \operatorname{Ctr}(M)$. To prove the converse, by Lemma 2.4 it is sufficient to show that there exists $n$ such that $\|\theta(v)-v\|_{2} \leq 12^{-8}, \forall v \in \mathcal{U}\left(N_{n}^{\prime} \cap M\right)$. Assume on the contrary that $\forall n, \exists v_{n} \in \mathcal{U}\left(N_{n}^{\prime} \cap M\right)$ such that $\left\|\theta\left(v_{n}\right)-v_{n}\right\|_{2}>12^{-8}$. But this implies that $v=\left(v_{n}\right) \in M^{\prime} \cap M^{\omega}$ satisfies $\theta(v) \neq v$, contradicting the central triviality of $\theta$.

2.7. Corollary. Assume the $\mathrm{II}_{1}$ factor $M$ is an inductive limit of subfactors $N_{n} \nearrow$ $M$ such that $N_{n}$ has spectral gap in $M$ and $N_{n}^{\prime} \cap M$ is a factor, $\forall n$.

1. If $\theta \in \operatorname{Ctr}(M) \cap \overline{\operatorname{Int}(M)}$, then for any large enough $n$, there exist $u, v \in \mathcal{U}(M)$ such that $\theta=\operatorname{Ad} u$ on $N_{n}$ and $\theta=\operatorname{Ad} v$ on $N_{n}^{\prime} \cap M$. Moreover, if for such an $n$ we denote $\theta^{\prime}=\operatorname{Ad} u^{*} \theta$, then for any $\varepsilon>0$, there exist $m \geq n$ and a non-zero partial isometry $w \in N_{n}^{\prime} \cap M$ such that $\theta(x) w=w x, \forall x \in N_{m}^{\prime} \cap M$, and $\|w-1\|_{2} \leq \varepsilon$.

2. If in addition $\mathcal{N}_{M}\left(N_{n}^{\prime} \cap M\right)$ acts innerly on $N_{n}^{\prime} \cap M$, then $\operatorname{Ctr}(M) \cap \overline{\operatorname{Int}(M)} /$ $\operatorname{Int}(M)$ naturally embeds into the group of automorphisms $\theta \in \operatorname{Aut}(M)$ which act trivially on $N_{n} \vee N_{n}^{\prime} \cap M$.

Proof. By Lemmas 2.5 and 2.6, for any large enough $n$ there exist $u, v \in \mathcal{U}(M)$ such that $\theta^{\prime}=\operatorname{Ad}\left(u^{*}\right) \theta$ is trivial on $N_{n}$ and equal to $\operatorname{Ad}(v)$ on $N_{n}^{\prime} \cap M$. The rest of (1) follows from Lemma 2.4. Under the additional assumption in (2), it follows that $\theta^{\prime}$ can be perturbed by an element in $\operatorname{Int}\left(N_{n}^{\prime} \cap M\right)$ so that it can act trivially on $N_{n} \vee N_{n}^{\prime} \cap M$.

\section{Deformation-RIGIDITy LEMma AND EXAMPles}

3.1. Lemma. Let $Q \subset M$ be an inclusion of factors and assume that $Q$ has spectral gap in $M$. If $N_{n} \subset M$ are von Neumann subalgebras such that $\lim _{n}\left\|E_{N_{n}}(x)-x\right\|_{2}=$ $0, \forall x \in M$, then for any $\varepsilon>0$, there exists $n$ such that $N_{n}^{\prime} \cap M \subset_{\varepsilon} Q^{\prime} \cap M$. 
Proof. Since $Q$ has spectral gap in $M$, by definition there exist $\delta>0$ and $u_{1}, \ldots, u_{m}$ $\in U(Q)$ such that if $x \in(M)_{1}$ satisfies $\left\|\left[u_{i}, x\right]\right\|_{2}<\delta, \forall i$, then $x \in_{\varepsilon} Q^{\prime} \cap M$. By the hypothesis, there exists $n$ such that $\left\|E_{N_{n}}\left(u_{i}\right)-u_{i}\right\|_{2} \leq \delta / 2, \forall i$. Thus, if $x \in N_{n}^{\prime} \cap M$, then

$$
\left\|\left[x, u_{i}\right]\right\|_{2} \leq 2\|x\|\left\|E_{N_{n}}\left(u_{i}\right)-u_{i}\right\|_{2} \leq \delta, \forall i
$$

implying that $x \in_{\varepsilon} Q^{\prime} \cap M$.

We next look for conditions which are sufficient for the assumptions in Corollary 2.7 to be satisfied and for which Lemma 3.1 thus applies. As in [15], we denote by $\mathcal{G}_{P, N}$ the standard invariant of an inclusion of factors with finite index $P \subset N$ and we denote by $\Gamma_{P, N}$ its standard graph.

3.2. Proposition. 1. If $N$ is a non-Gamma $\mathrm{II}_{1}$ factor and $S$ is an arbitrary finite factor, then $N$ has spectral gap in $M=N \bar{\otimes} S$. Moreover, any subfactor of finite index $P \subset N$ has spectral gap in $M$.

2. If $N$ has the property (T) and $M$ is a $\mathrm{II}_{1}$ factor containing $M$, then $N$ has spectral gap in $M$. In particular, if $P$ is a subfactor of finite index of a property (T) factor $N, P \subset N \subset N_{1} \subset \ldots$ is the Jones tower and $N_{\infty}$ is the associated enveloping algebra, then $N_{n}$ has spectral gap in $N_{\infty}, \forall n$.

3. If $N$ is non-Gamma, $P \subset N$ is a subfactor with finite depth, and $N_{n} \nearrow N_{\infty}$ is the associated tower and enveloping algebra, then $N_{n}$ has spectral gap in $N_{\infty}$, $\forall n$.

4. Let $\mathcal{G}$ be a standard $\lambda$-lattice, let $Q$ be a $\mathrm{II}_{1}$ factor and denote by $P=$ $M_{-1}^{\mathcal{G}}(Q) \subset M_{0}^{\mathcal{G}}(Q)=N$ the inclusion of $\mathrm{II}_{1}$ factors with standard invariant $\mathcal{G}$, as constructed in [15], 16], with $N_{n} \nearrow N_{\infty}$ the associated tower and enveloping algebra. Then $N_{n}$ has spectral gap in $N_{\infty}, \forall n$. Also, if $Q=L\left(F_{\infty}\right)$, then $P \simeq$ $N_{n} \simeq L\left(\mathbb{F}_{\infty}\right), \forall n$.

Moreover, in part $3, N_{n}^{\prime} \cap N_{\infty}$ is a factor $\forall n$. In turn, in parts 2 and $4, N^{\prime} \cap N_{\infty}$ is a factor if and only if the standard graph of $P \subset N$ is ergodic. This is the case if for instance $\mathcal{G}_{N, M}$ is strongly amenable or if $P \subset N$ has graph $A_{\infty}$, i.e. when $[N: P] \geq 4$ and the relative commutants $N^{\prime} \cap N_{n}$ are generated by the Jones projections (i.e. $\mathcal{G}_{P, N}$ is the so-called Temperley-Lieb standard lattice).

Proof. Part 1 is essentially due to Connes (see [3]), and part 2 is trivial. Part 4 is immediate by the proofs of 7.1 and 7.3 in [13. To prove part 3, note first that if $N \supset P \supset P_{1} \supset P_{2} \supset \ldots$ is a tunnel, then $P_{k}$ has spectral gap in $N$ and thus in $N \vee N^{\prime} \cap N_{\infty}$, for any $k$. Since $P \subset N$ has finite depth, so does $N \vee N^{\prime} \cap N_{\infty} \subset N_{\infty}$ (see e.g. [9] or [4) and there exists $k$ such that $P_{k}^{\prime} \cap N_{\infty}$ contains an orthonormal basis $\left\{m_{j}\right\}_{j}$ of $N_{\infty}$ over $N \vee N^{\prime} \cap N_{\infty}$. Thus, if $\xi \in N^{\prime} \cap L^{2}\left(N_{\infty}\right)^{\omega}$, then $\xi=\Sigma_{j} m_{j} \xi_{j}$, for some unique "coefficients" $\xi_{j}$ lying in $L^{2}\left(N \vee N^{\prime} \cap N_{\infty}\right)^{\omega}$. Since $\xi$ and $m_{j}$ commute with $P_{k}$, it follows that the $\xi_{j}$ commute with $P_{k}$ as well, so in fact all $\xi_{j}$ lie in $\left(P_{k}^{\prime} \cap N\right) \vee L^{2}\left(N^{\prime} \cap N_{\infty}\right)^{\omega}$. Altogether, $\xi \in N^{\prime} \cap L^{2}\left(P_{k}^{\prime} \cap N_{\infty}\right)^{\omega}=L^{2}\left(N^{\prime} \cap N_{\infty}\right)^{\omega}$.

\section{Calculations of $\chi(M)$ and an answer to Connes' Question}

4.1. Theorem. If $M=\bar{\otimes}_{k} Q_{k}$, with $Q_{k}$ a sequence of non-Gamma $\mathrm{II}_{1}$ factors, then $\operatorname{Ctr}(M) \cap \overline{\operatorname{Int}(M)}=\operatorname{Int}(M)$ (equivalently $\chi(M)=1$ ) and $M$ is McDuff but not $s$-McDuff.

Proof. By Corollary 2.7.2 we have $\chi(M)=1$ (this calculation was in fact already done in [2]). Assume $M=Q \bar{\otimes} R$ for some non-Gamma factor $Q$. Thus, $Q$ has 
spectral gap in $M$. Denote $N_{n}=\bar{\otimes}_{k \leq n} Q_{k}$. Then $N_{n} \nearrow M$ and, by Lemma 3.2 there exists $n$ such that $\bar{\otimes}_{k>n} Q_{k}=N_{n}^{\prime} \cap M \subset_{\varepsilon} Q^{\prime} \cap M=R$. By [18, this implies there exists an isomorphism of $\bar{\otimes}_{k>n} Q_{k}$, which is a non-amenable factor, into an amplification of $R$. Since $R$ is amenable, this is a contradiction.

From here on, we consider the following special case of inductive limits of factors: Let $P \subset N$ be a subfactor of finite Jones index, let $P \subset N \subset N_{1} \subset N_{2} \subset \ldots$ be its Jones tower and let $M=N_{\infty}=\overline{\bigcup_{n} N_{n}}$ be the associated enveloping $\mathrm{II}_{1}$ factor. Under the assumption that $N$ has spectral gap in $N_{\infty}$, we relate Connes' $\chi$-invariant of $N_{\infty}$ with Kawahigashi's $\chi$-invariant of the inclusion $N^{\prime} \cap N_{\infty} \subset P^{\prime} \cap N_{\infty}$ and use this to calculate $\chi\left(N_{\infty}\right)$ for many enveloping factors. In particular, this will provide more examples of factors with trivial $\chi$-invariant which are McDuff but not s-McDuff.

Thus, recall from [7] that if $S \subset R$ is an inclusion of finite von Neumann algebras, then

$$
\chi(R, S) \stackrel{\text { def }}{=} \overline{\operatorname{Int}(R, S)} \cap \operatorname{Ctr}(R, S) / \operatorname{Int}(R, S),
$$

where $\operatorname{Int}(R, S)$ is the group of inner automorphisms of $R$ implemented by unitaries in $S, \overline{\operatorname{Int}(R, S)}$ is its closure and $\operatorname{Ctr}(R, S)$ is the group of automorphisms of $R$ leaving $S$ invariant and acting trivially on $R^{\prime} \cap S^{\omega}$. We consider this invariant for inclusions of the form $S=N^{\prime} \cap N_{\infty} \subset P^{\prime} \cap N_{\infty}=R$, where $P \subset N$ is a (proper) subfactor of finite index and $N_{\infty}$ is its enveloping algebra, as above.

4.2. Theorem. If $N$ has spectral gap in $N_{\infty}$, then there is a natural isomorphism $\chi\left(N_{\infty}\right) \simeq \chi\left(P^{\prime} \cap N_{\infty}, N^{\prime} \cap N_{\infty}\right)$.

Proof. Note first that by [12, if $\sigma$ is an automorphism of $R=P^{\prime} \cap N_{\infty}$ leaving $S=N^{\prime} \cap N_{\infty}$ invariant, then there exists $u \in \mathcal{U}\left(N^{\prime} \cap N_{\infty}\right)$ such that $u \sigma\left(e_{1}\right) u^{*}=e_{1}$, where $e_{1}=e_{P}$. Thus, any element in $\overline{\operatorname{Int}(R, S)} / \operatorname{Int}(R, S)$ can be represented by an automorphism of the form $\sigma=\lim _{n} \operatorname{Ad}\left(u_{n}\right)$, with $u_{n} \in \mathcal{U}\left(N_{1}^{\prime} \cap N_{\infty}\right)$. Denote by $\mathcal{G} \subset \operatorname{Aut}(R, S)$ the group of automorphisms of this form. Denote also $\mathcal{G}_{0}=$ $\mathcal{G} \cap \operatorname{Ctr}(R, S)$. Let also $\mathcal{H} \subset \operatorname{Aut}\left(N_{\infty}\right)$ be the group of automorphisms in $\overline{\operatorname{Int}\left(N_{\infty}\right)}$ which act trivially on $N_{1}$ and $\mathcal{H}_{0}=\mathcal{H} \cap \operatorname{Ctr}\left(N_{\infty}\right)$.

If $\sigma \in \mathcal{G}$, then let $\psi=\Psi(\sigma)$ be the Banach limit $\psi(x) \stackrel{\text { def }}{=} \operatorname{Lim}_{\omega} u_{n} x u_{n}^{*}, x \in N_{\infty}$. Thus $\psi$ is a unital trace preserving a completely positive (c.p.) map on $N_{\infty}$, and by the choice of $u_{n}$, we have $\psi(x)=x$ for $x \in N, \psi\left(e_{1}\right)=e_{1}$ and $\psi(x)=\sigma(x)$, $x \in P^{\prime} \cap N_{\infty}$. Note now that if $\psi$ is a unital trace preserving c.p. map on a finite von Neumann algebra $N_{\infty}$, then the set $\mathcal{X} \subset N_{\infty}$ on which it is $\|\cdot\|_{2}$-isometric is a von Neumann subalgebra and $\psi$ is multiplicative on $\mathcal{X}$. Indeed, $\mathcal{X}$ coincides with the space of fixed points of the unital trace preserving c.p. map $\psi^{*} \psi$, which is well known to be a von Neumann algebra. Thus, $\psi_{\mid \mathcal{X}}: \mathcal{X} \rightarrow N_{\infty}$ is a unital trace preserving, $\|\cdot\|_{2}$-isometric c.p. map, implying that it is a von Neumann algebra ${ }^{*}$-morphism. Since in our case $\psi$ is isometric on $N, e_{1}, N^{\prime} \cap N_{\infty}$ and the algebra generated by $N, e_{1}$ and $N^{\prime} \cap N_{\infty}$ is $\|\cdot\|_{2}$-dense in $N_{\infty}$, it follows that $\mathcal{X}=N_{\infty}$. Thus, $\psi \in \operatorname{Aut}\left(N_{\infty}\right)$ and so, by the way it was defined, $\psi \in \overline{\operatorname{Int}\left(N_{\infty}\right)}$. Moreover, since $N$ has spectral gap in $N_{\infty}, N_{\infty}^{\prime} \cap N_{\infty}^{\omega}=N_{\infty}^{\prime} \cap\left(N^{\prime} \cap N_{\infty}\right)^{\omega}=R^{\prime} \cap S^{\omega}$. Thus, if $\sigma \in \mathcal{G}_{0}$, then $\psi \in \operatorname{Ctr}\left(N_{\infty}\right)$.

Conversely, if $\theta \in \mathcal{H}$, then define $\Phi(\theta)$ to be the restriction of $\theta$ to $R=P^{\prime} \cap N_{\infty}$. Note that by Lemma 2.5 (a), we have $\Phi(\theta) \in \mathcal{G}$. Also, if $\theta \in \mathcal{H}_{0}$, then $\Phi(\theta) \in \mathcal{G}_{0}$. Since we clearly have $\Phi \circ \Psi=i d$ and $\Psi \circ \Phi=i d$, the statement follows. 
Note that, for a special class of inclusions of factors $P \subset N$, the equality $\chi(N, P)=\chi\left(N_{\infty}\right)$ was already established in [23.

4.3. Theorem. Let $P \subset N$ be a subfactor of finite index. Assume $N$ has spectral gap in $N_{\infty}$ and $N^{\prime} \cap N_{\infty}$ is a factor (e.g. $N$ is non-Gamma and $P \subset N$ has finite depth).

(i) $N_{\infty}$ is s-McDuff if and only if $P \subset N$ is a "matricial" inclusion; i.e., it is of the form $P \subset M_{n}(P)$, for some $n$, or equivalently $N=P \vee\left(P^{\prime} \cap N\right)$.

(ii) If $P^{\prime} \cap N=\mathbb{C}$ and $\Gamma_{P, N}$ is strongly amenable and its canonical weight vectors $\vec{s}=\left(s_{k}\right)_{k}$, resp. $\vec{t}=\left(t_{l}\right)_{l}$, have distinct entries (i.e. $s_{k} \neq s_{k^{\prime}}$ for $k \neq k^{\prime}$ and $t_{l} \neq t_{l^{\prime}}$ if $\left.l \neq l^{\prime}\right)$, then $\chi\left(N_{\infty}\right)=1$.

Proof. To prove (i), assume $Q \bar{\otimes} R=N_{\infty}$. Let $R_{n} \nearrow R$ be an increasing sequence of finite dimensional subfactors exhausting $R$. By Lemma 3.1 there exists $n$ such that $N \subset_{1 / 4} Q \bar{\otimes} R_{n}$. Since $N^{\prime} \cap N_{\infty}$ is a factor, by Proposition 12 in [10], it follows that there exists a unitary element $u \in N_{\infty}$ such that $u N u^{*} \subset Q^{t}$, for some $t>0$. Thus, by replacing $Q$ by $u^{*} Q^{t} u$ and $R$ by $u^{*} R^{1 / t} u$, we may assume the decomposition $N_{\infty}=Q \bar{\otimes} R$ is such that $N \subset Q$. Hence, $R=Q^{\prime} \cap N_{\infty} \subset N^{\prime} \cap N_{\infty}$.

Now note that since $R$ splits off $N_{\infty}$, it also splits off $N^{\prime} \cap N_{\infty}$; i.e., if we denote $B=R^{\prime} \cap\left(N^{\prime} \cap N_{\infty}\right)$, then $N^{\prime} \cap N_{\infty}=R \bar{\otimes} B$. In particular, $B$ is a factor.

On the other hand, by applying Lemma 3.1 again, for any $\varepsilon>0$ there exists $n$ such that $Q \subset_{\varepsilon / 2} N_{n}$. Taking relative commutants, it follows that $N_{n}^{\prime} \cap N_{\infty} \subset_{\varepsilon} R$. Thus, with the notation in [18, if we take $\varepsilon<1$, then we get $N_{n}^{\prime} \cap N_{\infty} \prec N_{\infty} R$, and thus $R \bar{\otimes} B=N^{\prime} \cap N_{\infty} \prec_{N_{\infty}} R$ as well (because $N_{n}^{\prime} \cap N_{\infty} \subset N^{\prime} \cap N_{\infty}$ has finite index). In particular, this shows that $B$ must be a finite dimensional factor. Thus, by replacing if necessary $R$ by $R \bar{\otimes} B$, we may actually assume $N^{\prime} \cap N_{\infty}=R$. So $N^{\prime} \cap N_{\infty}$ splits off $N_{\infty}$. Similarly $P^{\prime} \cap N_{\infty}$ splits off $N_{\infty}$ as well, implying that $N^{\prime} \cap N_{\infty}$ splits off $P^{\prime} \cap N_{\infty}$. Thus, $N^{\prime} \cap N_{\infty} \subset P^{\prime} \cap N_{\infty}$ is matricial, in particular extremal, which in turn implies $\left(P^{\prime} \cap N_{\infty}\right)^{\prime} \cap N_{\infty}=P \subset N=\left(N^{\prime} \cap N_{\infty}\right)^{\prime} \cap N_{\infty}$ is matricial as well.

To prove (ii), note that by Theorem 4.1 we have $\chi\left(N_{\infty}\right)=\chi\left(P^{\prime} \cap N_{\infty}, N^{\prime} \cap\right.$ $\left.N_{\infty}\right)$. Note also that by part 4 of Theorem 1.6 in [14, any automorphism $\theta$ of $N^{\prime} \cap N_{\infty} \subset P^{\prime} \cap N_{\infty}$ either is implemented by a unitary $u \in P^{\prime} \cap N_{\infty}$ normalizing $N^{\prime} \cap N_{\infty}$ or is properly outer. If $u$ is not in $N^{\prime} \cap N_{\infty}$, then the canonical vector of $\Gamma_{N^{\prime} \cap N_{\infty}, P^{\prime} \cap N_{\infty}}$ would have an even vertex other than $*$ equal to 1 . By strong amenability, $P=\left(P^{\prime} \cap N_{\infty}\right)^{\prime} \cap N_{\infty} \subset\left(N^{\prime} \cap N_{\infty}\right)^{\prime} \cap N_{\infty}=N$, which implies the same is true for $\Gamma_{P, N}$ and its standard vector, contradicting the hypothesis.

Thus, either $\theta=\operatorname{Ad}(u)$ for some $u \in N^{\prime} \cap N_{\infty}$ or $\theta$ is properly outer on the inclusions $N^{\prime} \cap N_{\infty} \subset P^{\prime} \cap N_{\infty}$. But by part 6 of Theorem 1.6 in [14], any properly outer automorphism of $N^{\prime} \cap N_{\infty} \subset P^{\prime} \cap N_{\infty}$ is centrally free; i.e., it acts freely on $N^{\prime} \cap\left(P^{\prime} \cap N_{\infty}\right)^{\omega}=N_{\infty}^{\prime} \cap N_{\infty}^{\omega}$, a contradiction. Thus, $\chi\left(P^{\prime} \cap N_{\infty}, N^{\prime} \cap N_{\infty}\right)$ must be trivial, and so $\chi\left(N_{\infty}\right)=1$ as well.

4.4. Corollary. For each $2 \leq n \leq \infty$, the factor $L\left(\mathbb{F}_{n}\right) \bar{\otimes} R$ has a sequence of irreducible subfactors $M_{m}$ with distinct indices satisfying $\chi\left(M_{m}\right)=1$ and which are McDuff but not s-McDuff.

Proof. By [22], for each odd number $m \geq 5$ there exists a subfactor $P \subset N=L\left(\mathbb{F}_{n}\right)$ of index $4 \cos ^{2} \pi / m$ and standard graph equal to $A_{m-1}$. Moreover, subfactors with such a graph have weight vectors with distinct entries, $\overrightarrow{s_{m}}=\left(s_{m, k}\right)_{k}$ and the numbers $r_{m}=\Sigma_{k} s_{m, k}^{2}$ are distinct, for $m \geq 5$ (see e.g. [5] or [4]). Also, if we fix 
$m$ and denote by $N_{\infty}$ the enveloping factor of the inclusion $P \subset N$ as before and let $R=N^{\prime} \cap N_{\infty}$, then the inclusion of factors $L\left(\mathbb{F}_{n}\right) \vee R \subset N_{\infty}$ has index equal to $r_{m}$ (see e.g. [4]). By [6], there exists a subfactor $M_{m} \subset L\left(\mathbb{F}_{n}\right) \vee R$ such that $L\left(\mathbb{F}_{n}\right) \vee R \subset N_{\infty}$ is the basic construction of $M_{m} \subset L\left(F_{n}\right) \vee R$, and by [12, $M_{m}$ is an amplification of $N_{\infty}$. Thus, since by Theorem $4.3 N_{\infty}$ is not s-McDuff and $\chi\left(N_{\infty}\right)=1$, it follows that $M_{m}$ satisfies the same properties as well.

4.5. Theorem. Let $P \subset N$ be an inclusion of non-Gamma $\mathrm{II}_{1}$ factors with Temperley-Lieb standard invariant and either $[N: P]<4, \Gamma_{P, N}=A_{2 n}$ for some $n \geq 2$ or $[N: P] \geq 4, \Gamma_{P, N}=A_{\infty}$. Assume $N$ has spectral gap in $N_{\infty}$ (note that this is automatic in the case where $[N: P]<4)$. If $[N: P] \leq 4$, then $\chi\left(N_{\infty}\right)=1$; while if $[N: P]>4$, then $\chi\left(N_{\infty}\right)=\mathbb{T}$.

Proof. The case $[N: P] \leq 4$ is a consequence of 4.3.(ii). To prove the case $[N$ : $P]>4$, note that by [13], the inclusion of factors $\left(P^{\prime} \cap N_{\infty}\right)^{\prime} \cap N_{\infty}=\tilde{P} \subset \tilde{N}=$ $\left(N^{\prime} \cap N_{\infty}\right)^{\prime} \cap N_{\infty}$ is locally trivial. More precisely, there is an isomorphism $\sigma: p \tilde{N} p \simeq$ $(1-p) \tilde{N}(1-p)$, where $p \in \tilde{P}^{\prime} \cap \tilde{N}$ is a projection satisfying $\tau(p) \tau(1-p)=[N: P]^{-1}$ such that $\tilde{P}=\{x+\sigma(x) \mid x \in p \tilde{N} p\}$. Also, the enveloping factor of $\tilde{P} \subset \tilde{N}$ is equal to $N_{\infty}$ and $\tilde{N}$ has spectral gap in $N_{\infty}$ (because $N$ does). But then Corollary 2.7.1 applies, showing that any element in $\overline{\operatorname{Int}\left(N_{\infty}\right)} \cap \operatorname{Ctr}\left(N_{\infty}\right) / \operatorname{Int}\left(N_{\infty}\right)$ is represented by an automorphism $\theta$ of $N_{\infty}$ which acts trivially on $\tilde{N}$ and for which there exist a non-zero partial isometry $w \in \tilde{N}^{\prime} \cap N_{\infty}$ and some $m$ such that $\theta(x) w=w x$, $\forall x \in \tilde{N}_{m}^{\prime} \cap N_{\infty}$. Note that $w^{*} w$ lies in $\left(\tilde{N}_{m}^{\prime} \cap N_{\infty}\right)^{\prime} \cap\left(\tilde{N}^{\prime} \cap N_{\infty}\right)=\tilde{N}^{\prime} \cap \tilde{N}_{m}$.

By multiplying from the right on both sides with a minimal projection $q \leq w^{*} w$ in $\tilde{N}^{\prime} \cap \tilde{N}_{m}$, we may in fact assume $w^{*} w=q$. Since the minimal projections in distinct direct summands of $\tilde{N}^{\prime} \cap \tilde{N}_{m}$ have distinct traces, it follows that both $w w^{*}$ and $\theta(q)$ are minimal projections in the same direct summand of $\theta\left(\tilde{N}^{\prime} \cap \tilde{N}_{m}\right)$. Thus, by multiplying $w$ from the left with an appropriate partial isometry in $\theta\left(\tilde{N}^{\prime} \cap \tilde{N}_{m}\right)$, we may assume $w w^{*}=\theta(q)$. So, now we have $\theta(x) w=w x, \forall x \in\left(\tilde{N}_{m}^{\prime} \cap N_{\infty}\right) q=$ $q\left(\tilde{N}^{\prime} \cap N_{\infty}\right) q$. But this implies there exists a unitary element $v \in \tilde{N}^{\prime} \cap N_{\infty}$ such that $\theta=\operatorname{Ad} v$ on $\tilde{N}^{\prime} \cap N_{\infty}$. Altogether, this shows that any element in $\chi\left(N_{\infty}\right)$ can be represented by an automorphism acting trivially on $Q=\tilde{N} \vee \tilde{N}^{\prime} \cap N_{\infty}$; i.e., $\chi\left(N_{\infty}\right)$ naturally embeds into the group $G$ of automorphisms of $N_{\infty}$ acting trivially on $Q$.

But $N_{\infty}$ is isomorphic to the crossed product of $Q$ by an aperiodic automorphism, whose amplification to $Q \bar{\otimes} \mathcal{B}(\mathcal{H}) \bar{\otimes} \mathcal{B}(\mathcal{H})$ acts by $\sigma \otimes \sigma_{0}$, where $\sigma$ is an automorphism of $\tilde{N} \bar{\otimes} \mathcal{B}(\mathcal{H})$ scaling the trace by $t /(1-t)$ and $\sigma_{0}$ is an automorphism of $\tilde{N}^{\prime} \cap$ $N_{\infty} \bar{\otimes} \mathcal{B}(\mathcal{H}) \simeq R \bar{\otimes} \mathcal{B}(\mathcal{H})$ scaling the trace by $(1-t) / t$ (given by the inclusion $\tilde{N}^{\prime} \cap$ $\left.N_{\infty} \subset \tilde{P}^{\prime} \cap N_{\infty}\right)$. Thus $G=\mathbb{T}$, with each $\lambda \in \mathbb{T}$ corresponding to the automorphism $\theta_{\lambda}$ of $N_{\infty}=Q \rtimes \mathbb{Z}$ which leaves $Q$ pointwise fixed and satisfies $\theta_{\lambda}(u)=\lambda u$, where $u \in N_{\infty}$ is the canonical unitary implementing the action of $\mathbb{Z}$ on $Q$. To see that each $\theta_{\lambda}$ lies in $\overline{\operatorname{Int}\left(N_{\infty}\right)} \cap \operatorname{Ctr}\left(N_{\infty}\right)$, note that one can take a sequence of unitary elements $u_{n}$ in the hyperfinite $\mathrm{II}_{1}$ factor $R=\tilde{N}^{\prime} \cap N_{\infty}$ such that $\sigma_{0}\left(u_{n}\right)=\lambda u_{n}$ and $\left(u_{n}\right)_{n} \in R^{\prime} \cap R^{\omega}$. To see this, note that by Connes' classification of trace scaling automorphisms of the hyperfinite $\mathrm{II}_{\infty}$ factor $R^{\infty} \simeq \tilde{N}^{\prime} \cap N_{\infty} \bar{\otimes} \mathcal{B}(\mathcal{H})([1])$, we may assume $\tilde{N}^{\prime} \cap N_{\infty}=R_{1} \bar{\otimes} R_{2}$ and $\sigma_{0}=\sigma_{1} \otimes \sigma_{2}$, with $\sigma_{1}$ an automorphism of $R_{1} \bar{\otimes} \mathcal{B}(\mathcal{H})$ scaling the trace by $(1-t) / t$ and $\sigma_{2}$ an automorphism of $R_{2}=R^{\otimes \infty}$ which is an infinite tensor product $\sigma_{2}=\alpha^{\otimes \infty}$ of copies of an automorphism $\alpha$ of $R$ such that $\alpha(v)=\lambda v$, for some $v \in \mathcal{U}(R)$. Thus, if we take $u_{n}=1 \otimes 1 \otimes \ldots \otimes v \otimes 1 \ldots$, 
with $v$ on the $n$th position of the infinite tensor product, then $\theta=\lim _{n} \operatorname{Ad} u_{n}$ acts trivially on $Q$ while $\theta(u)=\lambda u$. Moreover, $\theta$ clearly belongs to $\overline{\operatorname{Int}\left(N_{\infty}\right)} \cap \operatorname{Ctr}\left(N_{\infty}\right)$ by the way it was defined.

\section{REFERENCES}

[1] A. Connes: Outer conjugacy classes of automorphisms of factors, Ann. Sci. École Norm. Sup. 8 (1975), 383-419. MR0394228 (52:15031)

[2] A. Connes: Sur la classification des facteurs de type II, C. R. Acad. Sci. Paris 281 (1975), 13-15. MR0377534 (51:13706)

[3] A. Connes: Classification of injective factors, Ann. of Math. (2) 104 (1976), 73-115. MR0454659 (56:12908)

[4] D. E. Evans, Y. Kawahigashi: "Quantum symmetries on operator algebras", Oxford University Press, 1998. MR1642584 (99m:46148)

[5] F. Goodman, P. de la Harpe, V.F.R. Jones: "Coxeter graphs and towers of algebras", MSRI Publications, 14, Springer-Verlag, 1989. MR999799 (91c:46082)

[6] V.F.R. Jones: Index for subfactors, Invent. Math. 72 (1983), 1-25. MR696688 (84d:46097)

[7] Y. Kawahigashi: Centrally trivial automorphisms and an analogue of Connes's $\chi(M)$ for subfactors, Duke Math. J. 71 (1993), 93-118. MR.1230287 (94k:46131)

[8] D. McDuff: Central sequences and the hyperfinite factor, Proc. London Math. Soc. (3) 21 (1970), 443461. MR0281018 (43:6737)

[9] A. Ocneanu: Quantized groups, string algebras and Galois theory for von Neumann algebras, in "Operator Algebras and Applications", London Math. Soc. Lect. Notes Series, Vol. 136, 1988, pp. 119-172. MR996454 (91k:46068)

[10] N. Ozawa, S. Popa: Some prime factorization results for type $\mathrm{II}_{1}$ factors, Invent. Math. 156 (2004), 223-234. MR2052608 (2005g:46117)

[11] N. Ozawa, S. Popa: On a class of $\mathrm{II}_{1}$ factors with at most one Cartan subalgebra, math.OA/0706.3623, to appear in Annals of Mathematics.

[12] M. Pimsner, S. Popa: Entropy and index for subfactors, Ann. Sci. École Norm. Sup. (4) 19 (1986), 57-106. MR860811 (87m:46120)

[13] S. Popa: Markov traces on universal Jones algebras and subfactors of finite index, Invent. Math. 111 (1993), 375-405. MR1198815 (94c:46128)

[14] S. Popa: Classification of actions of discrete amenable groups on amenable subfactors of type II, IHES preprint, 1992, to appear in Intern. J. Math, 2009 (see http://www.math.ucla.edu/popa/preprints.html).

[15] S. Popa, An axiomatization of the lattice of higher relative commutants of a subfactor, Invent. Math. 120 (1995), 427-445. MR.1334479 (96g:46051)

[16] S. Popa: Universal construction of subfactors, J. Reine Angew. Math. 543 (2002), 39-81. MR.1887878 (2002k:46163)

[17] S. Popa: On a class of type $\mathrm{II}_{1}$ factors with Betti numbers invariants, Ann. of Math. (2) 163 (2006), 809-899. MR2215135 (2006k:46097)

[18] S. Popa: Strong rigidity of $\mathrm{II}_{1}$ factors arising from malleable actions of w-rigid groups. I, Invent. Math. 165 (2006), 369-408. MR2231961 (2007f:46058)

[19] S. Popa: On the superrigidity of malleable actions with spectral gap, J. Amer. Math. Soc. 21 (2008), 981-1000. MR 2425177 (2009e:46056)

[20] S. Popa: On the classification of inductive limits of $\mathrm{II}_{1}$ factors with spectral gap, math.OA/0910.2241.

[21] S. Popa, D. Shlyakhtenko: Universal properties of $L\left(\mathbb{F}_{\infty}\right)$ in subfactor theory, Acta Mathematica 191 (2003), 225-257. MR2051399 (2005b:46140)

[22] F. Rădulescu: Random matrices, amalgamated free products and subfactors of the von Neumann algebra of a free group, of noninteger index, Invent. Math. 115 (1994), 347-389. MR.1258909 (95c:46102)

[23] F. Rădulescu: An invariant for subfactors of the von Neumann algebra of a free group, Proceedings of the Fields Institute Workshop in Free Probability, D. Voiculescu, ed., Amer. Math. Soc., 1997, pp. 213-239. MR.1426841 
[24] S. Popa: Classification of subfactors and their endomorphisms, CBMS Lecture Notes, 86, Amer. Math. Soc., 1995. MR1339767 (96d:46085)

[25] S. Popa: Deformation and rigidity for group actions and von Neumann algebras, in "Proceedings of the International Congress of Mathematicians" (Madrid, 2006), Volume I, EMS Publishing House, Zurich, 2006/2007, pp. 445-477. MR2334200(2008k:46186)

Department of Mathematics, University of California, los Angeles, Los Angeles, CALIFORnia 90095-1555

E-mail address: popa@math.ucla.edu 